A. S. Wan, R. Cauble, P. Celliers, L. B. Da Silva, S. B. Libby,

R. A. London, J. Nilsen, J. C. Moreno, F. Weber

This paper was prepared for submittal to the 1995 Institute for Electrical and Electronics Engineers

Lasers and Electro Optics Society Annual Meeting

San Francisco, CA

October 30-November 2, 1995

August 1995

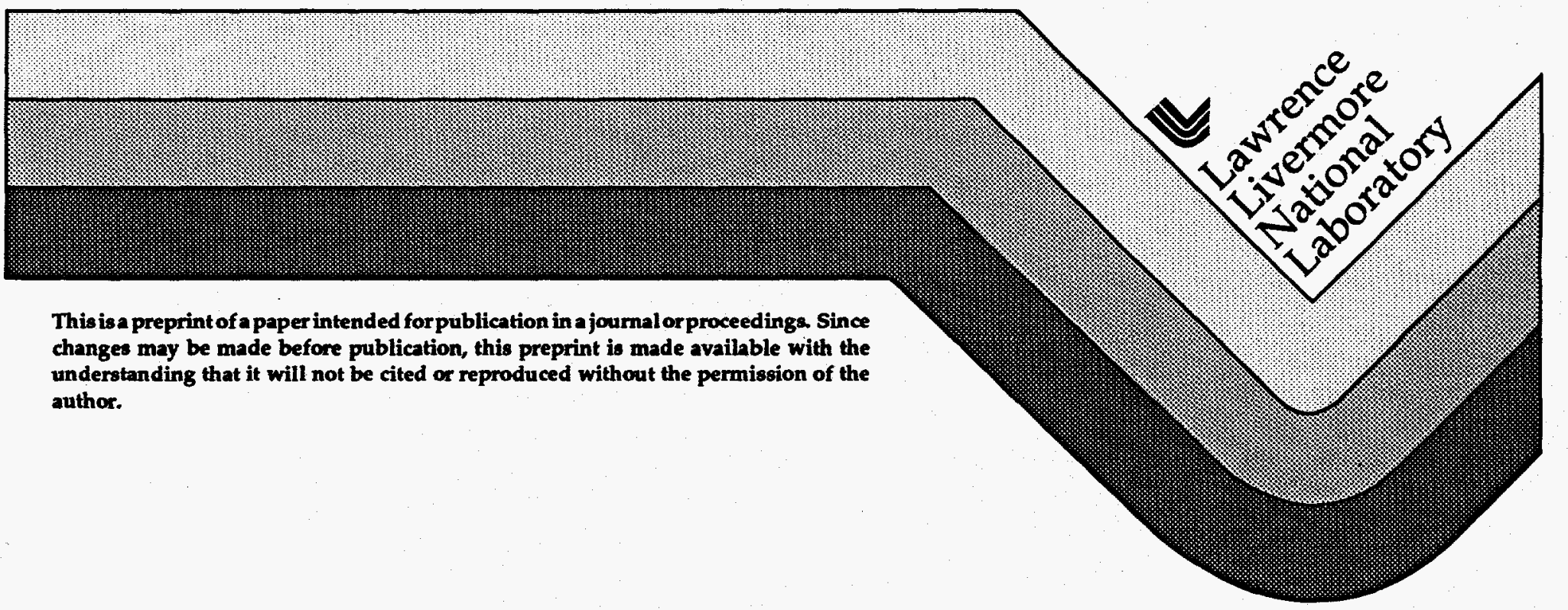




\section{DISCLAIMER}

This document was prepared as an account of work sponsored by an agency of the United States Government. Neither the United States Government nor the Uni versity of California nor any of their employees, makes any warranty, express or implied, or essumes any legal liability or responsibility for the accuracy, completeness, or usefulness of any information, apparatus, product, or process disclosed, or represents that its use would not infringe privately owned rights. Reference herein to any specific commercial products, process, or service by trade name, trademark, manufacturer, or otherwise, does not necessarily constitute or imply its endorsement, recommendation, or favoring by the United States Government or the University of California. The views and opinions of authors expressed herein do not necessarily state or reflect those of the United States Government or the University of California, and shall not be used for advertising or product endorsement purposes. 


\section{DISCLAIMER}

Portions of this document may be illegible in electronic image products. Images are produced from the best available original document. 


\title{
Characterization and Modeling of Soft X-ray Lasers
}

\author{
Alan S. Wan, Robert Cauble, Peter Celliers, Luiz B. Da Silva, Stephen B. Libby, \\ Richard A. London, Joseph Nilsen, Juan C. Moreno, Franz Weber
}

Lawrence Livermore National Laboratory, Livermore, CA 94550, U.S.A.

\begin{abstract}
This paper describes our theoretical, numerical, and experimental development of short-pulseduration, high brightness, and enhanced coherence x-ray lasers (XRLs) as sources suitable for applications as imaging diagnostics for laser plasmas.

\section{Introduction}

With its short wavelength ( $40-400 \AA$ ), short controllable pulse duration, high peak brightness, and sufficient spatial and temporal coherence, soft XRLs have been considered for applications in the fields of microscopy, holography, material science, and plasma physics. 1 Utilizing multilayer mirrors and beamsplitters and a collisionally pumped neon-like yttrium XRL operating at $155 \AA$ as the probe source, we have recently demonstrated a soft XRL interferometer in a skewed MachZehnder configuration. 2 With this soft XRL interferometer, we have measured two-dimensional electron density profiles of fast evolving, high-density laser-produced plasmas that exceed $10^{21}$ $\mathrm{cm}^{-3}$ for a large plasma that is millimeters in extent.
\end{abstract}

For plasma imaging applications we typically use a 3-cm-long yttrium slab XRL, ${ }^{3}$ driven by a 600 ps gaussian optical laser pulse, with an output energy of $\sim 8 \mathrm{~mJ}, \sim 300$-ps full-width at halfmaximum (FWHM) pulse duration, $\sim 100 \mu \mathrm{m}$ diameter source size, 10-mrad divergence, and bandwidth $(\lambda / \Delta \lambda)$ of $10^{4}$, which corresponds to a brightness of $\sim 10^{17} \mathrm{~W} / \mathrm{sr}-\AA-\mathrm{cm}^{2}$. This brightness is equivalent to a $6-\mathrm{GeV}$ blackbody, which, when used in conjunction with multilayer optics designed for the XRL wavelength, can overwhelm the self-emission of the target plasma. This paper describes our recent experimental and theoretical characterization and modeling of XRLs at the Lawrence Livermore National Laboratory (LLNL). These studies point toward methods to further improve the XRL performance for future plasma imaging applications.

\section{Properties of Soft X-ray Laser}

The coupling of the optical laser with the XRL target produced rapid plasma expansion with strong electron density gradient which resulted in large refraction and large beam divergence. By using either a prepulse or a multiple, picket-fence-shaped driving optical laser pulse, the main driving laser pulse is now coupled to a pre-formed plasma and lasing occurs at a region with flatter density profiles. With the new pulse shapes, we observed strong 3p - 3s J = 0-1 XRL output which dominates the XRL spectrum. Strong $J=0-1$ XRLs have now been observed over a large number of elements, ranging as low as chlorine at $\mathrm{Z}=17$ to selenium at $\mathrm{Z}=34.4 .5$ This reduced refraction is a likely explanation for the emergence of the $J=0-1$ line which lases at higher density regime. Another way to compensate for refraction is by bending the XRL target at an appropriate curvature. By proper guiding of the XRL photons, Kodama ${ }^{6}$ has observed a narrowing of the beam divergence to $\sim 1 \mathrm{mrad}$, which represents a significant increase in the output brightness.

We are developing techniques to shorten the time duration of the XRL while maintaining high brightness in order to optimize their usefulness as a plasma diagnostics. The drive pulse duration is directly related to the XRL duration. Using multiple pulse techniques and combined with traveling wave geometry, ${ }^{7}$ we have shortened the $x$-ray lasing duration down to $<50$ ps. However, the intensity of the XRL is about two orders of magnitude weaker as compared to long- 
duration pumping. We are looking at using curved targets and pulse shaping techniques ${ }^{8}$ to more efficiently pump the neon-like XRL system and to increase the XRL output.

We are also developing an XRL cavity to boost the output of XRLs while maintaining a shortpulse duration. Our approach on cavity development ${ }^{9}$ is to place the injection mirror far away from the XRL to minimize multilayer damage and to use the multiple-pulse configuration on Nova, timed such that the XRL photons produced in earlier pulses will be reinjected and propagate through the gain medium created by latter pulses. We verify the successful locking by measuring the near-field emission profiles of the XRL plasma, from which we can also estimate the spatial gain and $n_{e}$ profiles, and how they affect the dynamics of XRL plasmas.

The spatial and temporal coherence of the XRL become an important consideration in the design of a Mach-Zehnder interferometer. The observed fringe visibility is sensitive to both the temporal and spatial overlap of the recombined beams which allows us to characterize the spatial and temporal coherence of the XRL.10 By changing the optical path length between the two arms of the interferomenter and perform a gaussian fit to the measured fringe visibility, we obtained a $1 / e$ width of $100 \mu \mathrm{m}$ for the yttrium XRL line at $155 \AA$, which is approximately equivalent to a FWHM linewidth of $13 \mathrm{~m} \AA$. This is consistent with previous direct measurement of the linewidth using a high-precision spectrometer. ${ }^{11}$ We also measured an effective source dimension of $320 \mu \mathrm{m}$ by quantifying the change of the fringe visibility over a distorted region of the beamsplitter.

Beyond the 2-D interferometer, a diffraction-limited XRL can yield flash 3-D interferometric image of an ICF capsule. We are working on improving the spatial coherence of current XRLs by developing small-source-size oscillators and multiple-component XRL architectures. By controlling the shape of XRLs during plasma expansion and reducing the effective XRL source size using the concept of adaptive spatial filtering, we can obtain a diffraction-limited XRL with a high effective power. With the reduced source size, these XRLs can serve as driving oscillators in a multiple-component XRL architecture. Part of our effort are devoted to study the hydrodynamics and laser physics of such shaped XRLs and to study the coupling of multiple components in such laser architectures. 12

\section{Acknowledgments}

This work is performed under the auspices of the U. S. Department of Energy by LLNL under Contract W-7405-ENG-48.

\section{References}

1. See papers in Proc. of the Applications of X-ray Lasers Workshop, edited by R. A. London, D. L. Matthews, and S. Suckewer, LLNL Report CONF-9206170 (January 1992).

2. $\quad$ L. B. Da Silva, et al., Phys. Rev. Lett., 74, 3991 - 3994 (1995).

3. L. B. Da Silva, et al., Opt. Lett. 18, 1174 - 1176 (1993).

4. E. Fill et al., SPIE Proceeding "Soft X-ray Lasers and Applications," San Diego (1995)

5 J. Nilsen, et al., Phys. Rev. A, 48, 4682 - 4685 (1993).

$6 \quad$ R. Kodama, et al., Phys. Rev. Lett., 73, 3215 - 3218 (1994).

7. J. C. Moreno, et al., Opt. Commun., 110585 - 589 (1994)

8. J. C. Moreno, et al., SPIE Proceeding "Soft X-ray Lasers and Applications," San Diego (1995)

9. A. S. Wan, et al., "X-ray Laser 1994," AIP Conf. Proc. 332, D. Eder, D. Matthews, ed., Williamsburg, VA, 350-358 (May 1994)

10. P. Celliers, submitted to Opt. Lett.

11. J. A. Koch, et al., Phys. Rev. Lett., 68, 3291 (1992).

12. A. S. Wan, et al., Opt. Engrg., 33, $2434-2441$ (1994). 